BMJ Open

Diabetes

Research

\& Care

\title{
Seasonality of gestational diabetes mellitus: a South Australian population study
}

\author{
Petra E Verburg, ${ }^{1,2}$ Graeme Tucker, ${ }^{3,4}$ Wendy Scheil,, ${ }^{3,4}$ Jan Jaap H M Erwich, ${ }^{2}$ \\ Gus A Dekker, ${ }^{1,5}$ Claire T Roberts ${ }^{1}$
}

To cite: Verburg PE, Tucker G, Scheil W, et al. Seasonality of gestational diabetes mellitus: a South Australian population study. BMJ Open Diabetes Research and Care 2016;4:e000286. doi:10.1136/bmjdrc-2016000286

Received 21 June 2016 Revised 31 August 2016 Accepted 20 September 2016

\section{(a) CrossMark}

${ }^{1}$ Robinson Research Institute, Adelaide Medical School, University of Adelaide, Adelaide, South Australia, Australia

${ }^{2}$ Department of Obstetrics and Gynaecology, University Medical Center Groningen, University of Groningen, Groningen, The Netherlands ${ }^{3}$ Epidemiology Branch, SA Health, Adelaide, South Australia, Australia ${ }^{4}$ Adelaide Medical School, University of Adelaide, Adelaide, South Australia, Australia

${ }^{5}$ Department of Obstetrics and Gynaecology, Lyell McEwin Hospital, Elizabeth Vale, South Australia, Australia

Correspondence to Professor Claire T Roberts; claire.roberts@adelaide.edu.au

\section{ABSTRACT}

Objective: To investigate whether there is a seasonal variation in the incidence of gestational diabetes mellitus (GDM).

Research design and methods: This retrospective cohort study of 60306 eligible South Australian liveborn singletons during 2007-2011 recorded in the South Australian Perinatal Statistics Collection (SAPSC) examined the incidence of GDM in relation to estimated date of conception $(\mathrm{eDoC})$. Fourier series analysis was used to model seasonal trends.

Results: During the study period, $3632(6.0 \%)$ women were diagnosed with GDM. Seasonal modeling showed a strong relation between GDM and eDoC $(p<0.001)$. Unadjusted and adjusted models (adjusted for maternal age, body mass index (BMI), parity, ethnicity, socioeconomic status, and chronic hypertension) demonstrated the presence of a peak incidence occurring among pregnancies with $\mathrm{eDoC}$ in winter (June/July/August), with a trough for eDoc in summer (December/January/February). As this was a retrospective study, we could only use variables that had been collected as part of the routine registration system, the SAPSC.

Conclusions: This study is the first population-based study to demonstrate a seasonal variation for GDM. Several maternal lifestyle and psychosocial factors associated with seasonality and GDM may be influential in the pathophysiologic mechanisms of GDM. Ambient temperature, physical activity, nutrient intake, and vitamin $D$ levels may affect maternal physiology, and fetal and placental development at the cellular level and contribute to the development of GDM. The mechanisms underlying these possible associations are not fully understood and warrant further investigation.

\section{INTRODUCTION}

According to current guidelines, gestational diabetes mellitus (GDM) is defined as carbohydrate intolerance, with onset or first recognition during pregnancy. ${ }^{1}$ The incidence of GDM has increased several folds over the years from $1.9 \%$ in $1989-1990$ to $4.2 \%$ in 2003-2004. ${ }^{2}$ Currently, GDM is present in $\sim 7 \%$ of all pregnancies, varying from $1 \%$ to $10 \%$ depending on the studied population. ${ }^{1}$

\section{Significance of this study}

What is already known about this subject?

- Gestational diabetes mellitus (GDM) has important negative effects on maternal and offspring health but the pathophysiology is not fully understood. A seasonal variation in the incidence of GDM has been suggested, but it has never been shown in population-based studies before.

\section{What are the new findings?}

- This population-based study observed a clear seasonality in the incidence of GDM, with a peak incidence for pregnancies with conception in winter and the lowest incidence for pregnancies with conception in summer.

How might these results change the focus of research or clinical practice?

- Our data point to specific biological mechanism (s), such as maternal lifestyle choices and environmental factors, which may provide potential targets for the prevention of GDM and warrant further investigation.

The increase of GDM represents a significant economic burden on society, with direct medical costs of GDM in the USA in 2007 estimated at $\$ 636$ million. $^{3}$

GDM is an important contributor to maternal morbidity. Women with GDM have a 1.5-fold increased risk of hypertensive disorders of pregnancy, ${ }^{4}$ a 1.1 -fold risk of delivery by elective caesarean section, ${ }^{5}$ a 7.4 -fold risk of later type 2 diabetes (T2D) ${ }^{6}$ and a 2.2-fold increased long-term risk of cardiovascular disease $(\mathrm{CVD})^{7}$ compared with those women who had a normoglycemic pregnancy.

GDM has also important offspring health effects, causing fetal morbidity and mortality. In GDM, the mother and the fetus is hyperglycemic due to free transport of glucose through the placenta. The fetus is forced to increase its own insulin production. Increased maternal glucose levels in pregnancy have been associated with increased frequencies of preterm birth, birth injury, a 
5.0-fold increased risk of large for gestational age babies, a 2.2-fold increased risk of neonatal hypoglycemia, requirement for intensive neonatal care and hyperbilirubinemia. ${ }^{5}$ Pre-existing diabetes has been seen to be associated with increased frequencies of congenital abnormalities. ${ }^{9}$ In adulthood, offspring from mothers with GDM are at increased risk of developing obesity, impaired glucose tolerance, T2D, and CVD. ${ }^{8}$

The pathophysiology of GDM is not fully understood, but a number of risk factors have been implicated, such as maternal overweight and obesity, low maternal birth weight, race, increased maternal age, family history of T2D, history of previous fetal death, and previous birth of a macrosomic infant. ${ }^{10}{ }^{11}$ Also, the recurrence rate of GDM in subsequent pregnancies has shown to be as high as $47.2 \%{ }^{12}$

Other risk factors associated with GDM are nutrient intake, specifically high intake of saturated fat ${ }^{10}$ high ambient temperature, ${ }^{13} 14$ and a low maternal vitamin D status. $^{8}{ }^{15-22}$ Physical activity before and during pregnancy is associated with a significantly lower risk of developing GDM. ${ }^{23}$ Since some of these risk factors have shown a seasonality, ${ }^{24-26}$ we hypothesized that the incidence of GDM may differ over the seasons. A seasonal trend of GDM could provide more insight on its pathophysiology. So far, only one study has reported seasonal changes in the prevalence of GDM. ${ }^{27}$

In an effort to increase the knowledge of mechanisms regarding early pregnancy exposures that may influence the development of GDM, we aimed to assess the seasonal variation of GDM in a large population database. We focused on the estimated date of conception (eDoC) based on the hypothesis that the etiology of GDM lies early in pregnancy (and often prepregnancy), weeks to months before the clinical presentation of GDM.

\section{RESEARCH DESIGN AND METHODS}

\section{Study design and setting}

This was a retrospective cohort study investigating the incidence of GDM in relation to $\mathrm{eDoC}$ in South Australia (SA) during 2007-2011. The majority of the state's population lives within the metropolitan area of the state capital, Adelaide. Adelaide (situated $34^{\circ} \mathrm{S} 138^{\circ}$ E) is a coastal city with a Mediterranean climate, with long hot dry summers (December, January, February) and short cold rainy winters (June, July, August).

\section{Database}

Data were sourced from the South Australian Perinatal Statistics Collection (SAPSC), maintained by the Pregnancy Outcome Unit (POU) of SA Health. The SAPSC collects information regarding the characteristics and outcome of all births, of any gestation, and stillbirths and terminations of pregnancy of at least $400 \mathrm{~g}$ birth weight or 20 weeks of gestation, in South Australia. The data were notified by hospital and homebirth midwives, and neonatal nurses using a Supplementary Birth Record (SBR).

\section{Data selection}

The study included live-born singleton births in women with a known body mass index (BMI) with a gestation of 20-42 weeks and a birth weight $\geq 400 \mathrm{~g}$. Terminations of pregnancy and women with pre-existing diabetes were excluded from the analyses.

When the clinician documented that the woman had GDM, this was listed in the SAPSC. This was based on universal screening for GDM. All patients underwent a $50 \mathrm{~g}$ glucose challenge test at 28 weeks. Patients with an abnormal glucose challenge test $(\geq 7.8 \mathrm{mmol} / \mathrm{L})$ underwent a glucose tolerance test (GTT); fasting $\geq 5.5 \mathrm{mmol} / \mathrm{L}$ and/or 2-hour value $\geq 8 \mathrm{mmol} / \mathrm{L}$ were the constant cut-off values to diagnose GDM in 2007-2011 in South Australia.

The eDoC was estimated based on the birth date and gestational age at birth. Gestational age was determined by best obstetric estimate and reported as completed weeks. This estimate was based on the dating ultrasound (performed at 8-13 weeks gestation) supported by the known first day of the last menstrual period or by review of other ultrasonography. The database did not indicate how gestational age was specifically determined for each woman, but $99.1 \%$ of the women had an antenatal ultrasound.

\section{Variables}

The variables included were maternal age, BMI, parity, ethnicity, socioeconomic status (SES), civil status, smoking status at time of conception, and in the second half of pregnancy, chronic hypertension, asthma, and fetal sex. BMI was calculated before 20 weeks of gestation with the formula $\mathrm{BMI}=$ weight in $\mathrm{kg} /(\text { height in } \mathrm{m})^{2}$. Underweight was defined as $<18.5 \mathrm{~kg} / \mathrm{m}^{2}$, normal weight as $18.5-24.99 \mathrm{~kg} / \mathrm{m}^{2}$, overweight as $25.00-29.99 \mathrm{~kg} / \mathrm{m}^{2}$, obese as $30.00-39.99 \mathrm{~kg} / \mathrm{m}^{2}$, and morbidly obese $>40.00 \mathrm{~kg} / \mathrm{m}^{2}$. The Australian Bureau of Statistics' Socioeconomic Index for Areas (SEIFA) population-based quintile scores, derived from home address postcode, were used to indicate the SES. ${ }^{28}$ For the civil status the participants were divided into those with a stable partner (married/de facto) and those without a stable partner.

\section{Statistical analysis}

Data analysis was performed with SPSS V.21.0 (SPSS, 2013). Fisher's exact test and $\chi^{2}$ test were used to compare categorical variables. Differences were considered significant when the $\mathrm{p}$ value was $<0.05$.

In the primary analysis, eDoC was studied as a continuous variable using Fourier series methods (single cosinor analysis) ${ }^{29}$ Fourier series are considered to be the natural mathematic models for seasonality. To model the underlying seasonality of $\mathrm{eDoC}$, the first $\mathrm{p}$ pairs of term of the Fourier series was employed:

$$
\mathrm{S}\left(\Theta_{\mathrm{i}, \mathrm{p}}\right)=\sum_{\mathrm{r}=1}^{\mathrm{p}}\left\{\beta_{\mathrm{r}} \sin \left(\mathrm{r} \Theta_{\mathrm{i}}\right)+\gamma_{\mathrm{r}} \cos \left(\mathrm{r} \Theta_{\mathrm{i}}\right)\right\}
$$


In this series, $\Theta_{i}$ is the point in the annual cycle that the $\mathrm{i}^{\text {th }}$ day on which eDoC occurred. Denoting the number of days between 1 January 1950 and the $\mathrm{i}^{\text {th }}$ day of woman's eDoC as $D_{i}$, we calculated this angle in radians; thus, $\Theta_{\mathrm{i}}=2 \pi\left(\mathrm{D}_{\mathrm{i}} \bmod 365.25\right) / 365.25$. Thus, seasonal effect of the eDoC on the binary pregnancy outcome is modeled by adding $S\left(\Theta_{i}, p\right)$ to the linear predictor of a logistic regression model so that $\beta_{\mathrm{i}}$ and $\gamma_{\mathrm{i}}$ become parameters in a simple linear model. In these data, the first pair of Fourier terms (F1 model: sine and cosine) was significant based on a likelihood ratio test $(\alpha=0.10)$, allowing us to use these in this study.

Seasonality of GDM was initially investigated using a univariate logistic regression analysis using eDoC divided in months as predictor. Subsequently, the model was run with and without adjustment for potential confounders defined a priori (maternal age, BMI, parity, ethnicity, SES, civil status, smoking status, chronic hypertension, asthma, and fetal sex) using effects coding. The average of all months together was the reference in the model when using effects coding.

Subsequently, multivariate logistic regression models (Fourier series analysis) were fit by specifying a full model with all available data on potential modifiers and confounding variables defined a priori (see above). Covariates were considered not to be influential and were removed from the model via backward elimination if their contribution to the model was not significant $(p>0.05)$. Coefficient correlation tests were performed after every step of the model fitting process to test the goodness of fit.

\section{Ethics}

The existence of personal identifying information in the SAPSC was eliminated from the data file for analysis to ensure that confidentially of all patient records was maintained. The study protocol was approved by the Human Research Ethics Committee of the South Australian Department of Health (HREC/13/SAH/97).

\section{RESULTS}

\section{Study population}

Of a total 99973 births registered between January 2007 and December 2011, $60306(60.3 \%)$ births were eligible for the study. We excluded $25(0.03 \%)$ births with a gestational age of $<20$ weeks or $>42$ weeks, $269(0.27 \%)$ births in which the neonate had a birth weight $<400 \mathrm{~g}$, $121(0.12 \%)$ terminations of pregnancy because of chromosomal and/or congenital anomalies, 357 $(0.36 \%)$ stillbirths, 2961 (2.96\%) non-singleton births, $35542(35.55 \%)$ births with inadequately recorded maternal BMI, and $392(0.39 \%)$ births in which pregnancy was complicated by pre-existing diabetes.

\section{Incidence of GDM}

During the study period, a total of 3632 (6.0\%) women were diagnosed with GDM. The incidence of GDM increased significantly over the years from $4.9 \%$ in 2007 to $7.2 \%$ in $2011(\mathrm{p}<0.001$ (table 1$))$. The characteristics of the study population are presented in table 2 . Compared with non-GDM pregnancies, risk factors for GDM included age $>30$ years, multiparity, increased BMI, Asian ethnicity, low and very low SES, and a history of chronic hypertension.

\section{Seasonal models of GDM}

The observed, unadjusted and adjusted probabilities for GDM by eDoC are shown in figure 1. Both unadjusted and adjusted predicted probabilities were based on the logistic regression model fitting the first pair of Fourier series terms and showed that GDM was significantly related to season of eDoC $(\mathrm{p}<0.001)$.

The highest incidence of GDM was observed among pregnancies with eDoC in June (6.74\%). Unadjusted seasonal modeling showed peak incidence of GDM for pregnancies with eDoC in July $(6.55 \%)$, while adjusted seasonal modeling showed a peak incidence of GDM for pregnancies with eDoC in August (6.60\%).

The lowest incidence of GDM was observed among pregnancies with eDoC in January $(5.17 \%)$. Seasonal modeling showed a corresponding pattern. Unadjusted and adjusted predicted incidences showed a nadir for pregnancies with eDoC in January $(5.46 \%$ and $5.41 \%$, respectively).

\section{CONCLUSIONS}

\section{Increasing incidence of GDM}

The incidence of GDM in SA increased significantly from $4.9 \%$ in 2007 to $7.2 \%$ in 2011 . This is consistent with the trend in the USA, where incidence increased from $1.9 \%$ in $1989-1990$ to $4.2 \%$ in $2003-2004 .^{2}$ This increase in incidence is not surprising giving the parallel rise in prevalence of T2D and obesity ${ }^{8}$ and also increased focus on detection of GDM in pregnancy in recent decades.

\section{Seasonality of GDM}

In this large South Australian birth cohort, we observed a clear seasonality in the incidence of GDM based on eDoC. The peak incidence was observed for pregnancies with eDoC during winter and the lowest incidence for pregnancies with eDoC during summer.

\begin{tabular}{lccc} 
Table 1 & Incidence of GDM 2007-2011 & \\
\hline & \multicolumn{2}{l}{$\begin{array}{l}\text { Incidence GDM } \\
\text { complicated births }\end{array}$} & All births \\
\cline { 2 - 4 } Year of birth & $\mathbf{N}$ & Per cent & N \\
\hline 2007 & 396 & 4.9 & 8001 \\
2008 & 600 & 5.4 & 11062 \\
2009 & 780 & 6.0 & 12947 \\
2010 & 795 & 5.8 & 13633 \\
2011 & 1061 & 7.2 & 14663 \\
\hline
\end{tabular}

GDM, gestational diabetes mellitus. 
Table 2 Characteristics of the study population

\begin{tabular}{|c|c|c|c|c|c|c|}
\hline \multirow[b]{2}{*}{ Characteristic } & \multicolumn{2}{|c|}{$\begin{array}{l}\text { GDM complicated } \\
\text { births }\end{array}$} & \multicolumn{2}{|c|}{ Non-GDM births } & \multicolumn{2}{|c|}{ All births } \\
\hline & $\mathbf{n}$ & Per cent & $\mathbf{n}$ & Per cent & $\mathbf{n}$ & Per cent \\
\hline \multicolumn{7}{|l|}{ Age (in years) } \\
\hline$<20$ & 45 & 1.2 & 2653 & 4.7 & 2698 & 4.5 \\
\hline $20-25$ & 288 & 7.9 & 9941 & 17.5 & 10229 & 17.0 \\
\hline $25-30$ & 876 & 24.1 & 17593 & 31.0 & 18469 & 30.6 \\
\hline 30-35 & 1265 & 34.8 & 16480 & 29.1 & 17745 & 29.4 \\
\hline $35-40$ & 876 & 24.1 & 8298 & 14.6 & 9174 & 15.2 \\
\hline$>40$ & 281 & 7.7 & 1710 & 3.0 & 1991 & 3.3 \\
\hline \multicolumn{7}{|l|}{ BMI } \\
\hline$<18.5$ Underweight & 61 & 1.7 & 1837 & 3.2 & 1898 & 3.1 \\
\hline 18.5-25 Normal weight & 1091 & 30.0 & 27022 & 47.7 & 28113 & 46.6 \\
\hline 25-30 Overweight & 923 & 25.4 & 15217 & 26.8 & 16140 & 26.8 \\
\hline 30-40 Obese & 1155 & 31.8 & 10675 & 18.8 & 11830 & 19.6 \\
\hline >40 Morbid obese & 401 & 11.0 & 1924 & 3.4 & 2325 & 3.9 \\
\hline \multicolumn{7}{|l|}{ Parity } \\
\hline Nulliparous & 1452 & 40.0 & 24775 & 43.7 & 26227 & 43.5 \\
\hline Multiparous & 2179 & 60.0 & 31900 & 56.3 & 34079 & 56.5 \\
\hline \multicolumn{7}{|l|}{ Ethnicity } \\
\hline Caucasian & 986 & 27.2 & 9069 & 16.0 & 10055 & 16.7 \\
\hline Aboriginal or TSI & 94 & 2.6 & 1426 & 2.5 & 1520 & 2.5 \\
\hline Asian & 695 & 19.1 & 5186 & 9.2 & 5881 & 9.8 \\
\hline Other & 197 & 5.4 & 2457 & 4.3 & 2654 & 4.4 \\
\hline \multicolumn{7}{|l|}{ Socioeconomic status } \\
\hline Very low (0-20\%) & 1074 & 29.6 & 14660 & 25.9 & 15734 & 26.1 \\
\hline Low (20-40\%) & 786 & 21.6 & 12020 & 21.2 & 12806 & 21.2 \\
\hline Medium (40-60\%) & 695 & 19.1 & 11811 & 20.8 & 12506 & 20.7 \\
\hline High $(60-80 \%)$ & 549 & 15.1 & 9352 & 16.5 & 9901 & 16.4 \\
\hline Very high (<80-100\%) & 505 & 13.9 & 8621 & 15.2 & 9126 & 15.1 \\
\hline \multicolumn{7}{|l|}{ Civil status } \\
\hline With stable partner & 3296 & 90.8 & 49905 & 88.1 & 53201 & 88.2 \\
\hline Without stable partner & 335 & 9.2 & 6770 & 11.9 & 7105 & 11.8 \\
\hline \multicolumn{7}{|l|}{ Smoking } \\
\hline At time of conception $†$ & 633 & 17.4 & 11314 & 20.0 & 11947 & 19.8 \\
\hline Second half of pregnancy $\ddagger$ & 403 & 11.1 & 7780 & 13.7 & 8183 & 13.6 \\
\hline \multicolumn{7}{|l|}{ Medical complications } \\
\hline Chronic hypertension & 121 & 3.3 & 546 & 0.9 & 667 & 1.1 \\
\hline Asthma & 270 & 7.4 & 3643 & 6.4 & 3913 & 6.4 \\
\hline \multicolumn{7}{|l|}{ Fetal sex } \\
\hline Male & 1737 & 47.8 & 27631 & 48.7 & 29368 & 48.7 \\
\hline Female & 1895 & 52.1 & 29044 & 51.2 & 30939 & 51.3 \\
\hline
\end{tabular}

So far, only one study reported a seasonal pattern in the prevalence of GDM. ${ }^{27}$ The study carried out in Wollongong (coastal city, $34^{\circ} \mathrm{S}$ ), Australia, in 2010-2014, analyzed the results of 7343 oral GTTs performed between 24 weeks and 28 weeks gestation, and a total of $516(7.0 \%)$ women were diagnosed with GDM. The study found that in winter the median 1-hour and 2-hour glucose results were significantly lower compared with the overall 1-hour and 2-hour results. A post hoc analysis indicated that the prevalence of GDM based on the 1-hour and 2-hour results was higher in summer (4.6\% and $4.4 \%$, respectively) and lower in winter $(2.6 \%$ and $2.4 \%$, respectively) compared with the overall prevalence of GDM. Interestingly, they did not find a seasonal variation in fasting glucose levels. Diagnosis in summer corresponds with eDoC in winter, which concurs with our data.

Two previous studies reported that there was no seasonal pattern in the incidence of GDM. ${ }^{30}{ }^{31}$ An older and small study, carried out by the same group in Wollongong, Australia, in 1993-1994, analyzed a total of 2749 participants with an overall incidence of GDM of $6.7 \%$ (183 subjects). The seasonal fluctuation of GDM showed an incidence of $7.4 \%(5.6-9.2)$ in autumn 
Figure 1 Fitted seasonality for gestational diabetes mellitus (GDM) by estimated date of conception (eDoC). The histogram represents the observed probability of GDM by eDoC (divided in calendar months). The dashed line represents the unadjusted Fourier fit for the incidence of GDM by eDoC. The bold line represents the adjusted Fourier fit for the incidence of GDM by eDoC. The adjusted Fourier fit was adjusted for maternal age, BMI, parity, ethnicity, socioeconomic status, and chronic hypertension.

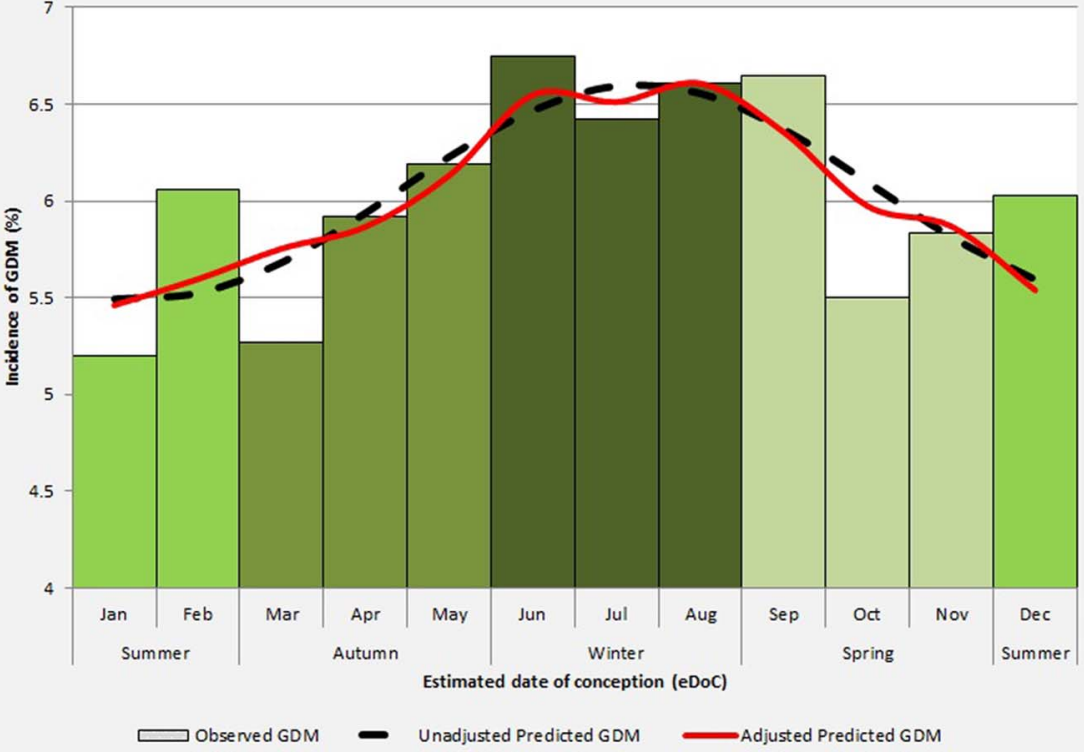

(March-May), corresponding with a conception in late winter/early spring (August-October). An incidence of $5.5 \%(3.5-7.5)$ was observed in winter, corresponding with a conception in late spring/early summer (November-January). However, this seasonal fluctuation was not statistically significant. ${ }^{30}$

Another small study was conducted in Plymouth (coastal city, $50^{\circ} \mathrm{N}$ ), UK, in 1996-1997. A total of 4942 pregnant women were studied, with an overall prevalence of GDM of $1.8 \%$ (90 women). The incidence of GDM ranged from $2.3 \%$ (1.5-3.2) in spring, corresponding with conception in autumn and the lowest incidence of GDM of $1.4 \%(0.8-2.3)$ in winter, corresponding with conception in summer. However, this seasonal fluctuation was also not statistically significant. ${ }^{31}$

Small sample size (183 and 90 women with GDM, respectively) and therefore lack of statistical power is the most likely reason due to which these two prior small studies could not detect significant seasonality. Also, Plymouth has a very mild climate, with little fluctuation in temperature and sun exposure, and therefore seasonality is expected to be less distinct than in more extreme climates. The actual trends were similar to the findings in this large South Australian cohort. Inconsistencies in the magnitude of GDM incidence are not surprising in this type of study, since population composition, environmental exposures, and climates differ in different regions of the world.

\section{Seasonality of type 2 diabetes}

Previous studies have analyzed the seasonal variation in T2D. A Hungarian study, investigating 26695 cases of T2D from 1999 to 2004 in Csongrad County, Hungary $\left(46^{\circ} \mathrm{N}\right)$, demonstrated a sinusoidal pattern for the incidence of T2D in studied males and females, with a peak incidence in spring and a trough in summer. ${ }^{32}$ This finding in T2D diagnosis is not in line with what we found in our GDM data. However, there are multiple potential reasons for this apparent discrepancy. Standardized testing in pregnancy between 24 and 28 weeks gestation eliminates patient's and doctor's delay in diagnosing GDM, versus T2D. Also, even though T2D and GDM are associated, the timelines to diagnosis are quite different.

Considering the Developmental origins of health and disease concept, ${ }^{33} 34$ suggesting prenatal programming of physiology, it can be hypothesized that T2D might show a seasonal variation depending on month of birth of the individual. Several studies have investigated this relationship with conflicting results. ${ }^{35} 36$ In an Ukranian study in three regions Charnigov $\left(51^{\circ} \mathrm{N}\right)$, Kherson $\left(46^{\circ} \mathrm{N}\right)$ and Rivne $\left(50^{\circ} \mathrm{N}\right)$ of 17510 male and 34704 female, type 2 diabetic patients showed a seasonal pattern with a peak in births in April for males and females, and a nadir of births for males in November and for females in December. ${ }^{35}$ However, a large Danish cohort study, investigating 223099 people, of whom 12468 developed T2D, did not find a relationship between season of birth and the incidence of T2D. ${ }^{36}$ However, as this study used time of birth to indicate season, it did not account for the fact that there was a range of gestational ages from preterm to term, possibly leading to confounding.

\section{Possible mechanisms underlying the seasonality of GDM}

In our analyses, we observed a seasonal variation of GDM after adjusting for maternal age, BMI, parity, ethnicity, SES, and chronic hypertension. These characteristics change relatively little from month to month and are not or barely modifiable. Owing to the retrospective character of the present study we could not adjust for several other meteorological, maternal lifestyle and psychosocial factors associated with seasonality, such as ambient temperature, physical activity, ${ }^{24}$ nutrient 
intake $^{25}$ and vitamin D status. ${ }^{26}$ Several studies have shown that these factors have been associated with the risk of GDM. ${ }^{1321233738}$

\section{Meteorological factors}

Studies suggest that the incidence of GDM can be associated with variable seasonal meteorological factors, specifically ambient temperature. A study in Porto Alegre (temperate climate $30^{\circ} \mathrm{S}$ ), Brazil, of 1030 pregnant individuals compared serum glucose in GTTs performed between 24 and 28 weeks gestation with ambient temperature. For every degree increase in ambient temperature, serum glucose increased by $0.07 \mathrm{mmol} / \mathrm{L}$. ${ }^{13}$ The Brazilian study suggest that the incidence of GDM may be highest in summer. GDM is generally diagnosed at 24-28 weeks of gestation, ${ }^{39}$ which corresponds with conception in winter. This is consistent with our findings that the highest incidence was seen when eDoC occurred in winter.

\section{Physical activity}

A systematic review and meta-analysis of 34929 participants and 2813 cases of GDM showed that prepregnancy and early pregnancy physical activity were significantly protective against GDM (55\% and 24\%, respectively, lower risk of GDM). ${ }^{23}$ During pregnancy, the body is subject to metabolic changes, especially during the third trimester, when GDM is typically diagnosed. GDM is thought to reflect an impaired capacity of the woman to adjust to these metabolic changes, due to relative $\beta$-cell dysfunction, that is, insulin secretion not keeping up with the exaggerated state of insulin resistance, resulting in abnormal glucose tolerance. An inverse association is plausible, and it can be suggested that prepregnancy and early pregnancy exercise may improve overall fitness and glucose tolerance. ${ }^{23}$

A systematic review has shown seasonal variation in the levels of physical activity in nonpregnant study populations. In general, the highest levels of physical activity were seen in spring and summer, while the lowest levels were seen in winter. ${ }^{24}$

These results are also consistent with our findings that the lowest incidence of GDM occurred with summer conceptions when women were more likely to be physically active. However, we are not aware of the studies reporting seasonal variation in levels of activity in pregnancy.

\section{Nutrient intake}

Seasonal variation of nutrient intake may affect metabolism, and this may also provide clues to understand the seasonal variation of GDM. Prospective ${ }^{25}$ and retrospective $^{40}$ studies have demonstrated a seasonal trend in nutrient intake in pregnant women. Unfortunately, data are not completely consistent. Previous studies have shown that a high total fat intake at time of diagnosis, in most cases at 24-28 weeks gestation, is associated with an increased risk for GDM. ${ }^{10} 37$ Interestingly, the total fat intake in one study was shown to be highest in spring, ${ }^{25}$ whereas the other showed the highest fat intake in summer. ${ }^{40}$ The latter corresponds to conception in autumn and winter, which is consistent with our findings.

\section{Vitamin D}

Serum vitamin D levels have a seasonal variation, with highest concentrations in summer and autumn and lowest concentrations in winter and spring. ${ }^{26}$ This is not surprising, since vitamin D is mainly derived from skin exposure to sunlight and dietary intake. ${ }^{41}$ The lowest dietary intake of vitamin $\mathrm{D}$ was seen in autumn and the highest in spring. ${ }^{25} 40$

A recent meta-analysis of studies published between 2008 and 2015 with over 9200 women showed that low vitamin $\mathrm{D}$ status associates with GDM. ${ }^{42}$ A vitamin D response element in the human insulin receptor gene promoter has been identified, ${ }^{43}$ suggesting a potential role for vitamin $\mathrm{D}$ in enhancing insulin secretion. Wang et $a l^{44}$ demonstrated that higher vitamin D levels stimulate increased $\beta$-cell mass and as such potentially also $\beta$-cell insulin secretion capacity. However, other studies have reported conflicting results. The association between vitamin $\mathrm{D}$ and its specific role in the development of GDM is contentious and merits further investigation.

\section{Strengths and weaknesses of the study}

A major strength of this population-based study lies in the large number of analyzed births. The SAPSC collects information regarding the characteristics and outcome of all births in SA, notified by hospital and homebirth midwives, and neonatal nurses.

As this was a retrospective study, we could only use variables that were already listed on the data set. GDM was listed in the SAPSC when the clinician documented that the women had GDM. In the study period, there were stable criteria for diagnosis and a consistent approach used by all care providers. This study included only the live-born babies during the study period. However, third trimester stillbirth is not very common, so it is unlikely that this has influenced the seasonal variation of GDM observed in this study. Since 2007, SAPSC began collecting data on BMI of the pregnant women. We used BMI as one of our inclusion criteria, which meant we had to exclude $35.55 \%$ of the total births between January 2007 and December 2011. SAPSC validation studies have shown that notifications of births in SA made by hospital and homebirth midwives, and hospital neonatal nurses on the SBR were robust for the parameters studied. ${ }^{45}$ The pregnancies analyzed in this study could therefore be considered as a proper representation of the South Australian and Australian population.

\section{Conclusion and further research}

This representative study has identified a statistically significant seasonal variation in the incidence of GDM. 
The etiology of GDM involves exposures that occur before it is clinically recognized. This suggests that the pathophysiologic mechanisms explaining (the seasonality of) GDM may have their origin early in pregnancy. These epidemiological data also support exploring the contributions of exposures with seasonal periodicity to GDM, such as environmental and lifestyle differences.

The effect size of seasonality of GDM presented in this paper is statistically significant, but unlikely to have direct clinical implications. However, our data point to specific biological mechanism(s) that warrant further investigation that may provide potential targets for the prevention of GDM. In particular, exposure(s) early in pregnancy deserve attention. These may include season of conception and vitamin D.

Acknowledgements The authors thank the midwives and staff of the Pregnancy Outcome Unit of South Australia Health for their passion and effort in managing and maintaining the completeness of the registry.

Contributors PEV and CTR are the guarantors of the work as a whole, including study design, access to the data, and the decision to submit and publish the manuscript. All authors have seen and approved the final version of this manuscript on submission. PEV has designed and executed the study, contributed to data analyses, interpreted the data, wrote the final manuscript and the critical discussion. GT contributed to study execution, carried out all necessary data analyses, and participated in interpretation of the data. WS contributed to study execution, data interpretation, and provided significant revisions. JJHME contributed to manuscript drafting and provided significant revisions. CTR participated in interpretation of the data, contributed in the critical discussion, and provided significant revisions. GAD contributed to study design, data interpretation, manuscript drafting, and critical discussion.

Funding PEV holds a PhD scholarship from the University of Groningen, Groningen, The Netherlands and a stipend from the Robinson Research Institute, University of Adelaide, Adelaide, Australia. CTR is a Senior Research Fellow (GNT1020749) at National Health and Medical Research Council of Australia (NHMRC) and is supported by a NHMRC project grant (GNT1059120).

\section{Competing interests None declared.}

Ethics approval Human Research Ethics Committee of the South Australian Department of Health.

Provenance and peer review Not commissioned; internally peer reviewed.

Data sharing statement The data were made available for this research by the South Australian Government Health Department, under ethics approval with strict no disclosure provisions for individual health records. Those wanting access to patient records for research purposes are advised to approach Dr Wendy Scheil, Pregnancy Outcome Unit, Epidemiology Branch, Department of Health, 11 Hindmarsh Square, Adelaide, South Australia (email Wendy.Scheil@ sa.gov.au, phone +61882266357).

Open Access This is an Open Access article distributed in accordance with the Creative Commons Attribution Non Commercial (CC BY-NC 4.0) license, which permits others to distribute, remix, adapt, build upon this work noncommercially, and license their derivative works on different terms, provided the original work is properly cited and the use is non-commercial. See: http:// creativecommons.org/licenses/by-nc/4.0/

\section{REFERENCES}

1. American Diabetes Association. Diagnosis and classification of diabetes mellitus. Diabetes Care 2014;37(Suppl 1): S81-90.

2. Getahun D, Nath C, Ananth CV, et al. Gestational diabetes in the United States: temporal trends 1989 through 2004. Am J Obstet Gynecol 2008;198:525.e1-5.
3. Chen Y, Quick WW, Yang W, et al. Cost of gestational diabetes mellitus in the United States in 2007. Popul Health Manag 2009;12:165-74.

4. Bryson CL, loannou GN, Rulyak SJ, et al. Association between Gestational Diabetes and Pregnancy-induced Hypertension. Am J Epidemiol 2003;158:1148-53.

5. Metzger BE, Lowe LP, Dyer AR, et al. Hyperglycemia and adverse pregnancy outcomes. N Engl J Med 2008;358:1991-2002.

6. Bellamy L, Casas JP, Hingorani AD, et al. Type 2 diabetes mellitus after gestational diabetes: a systematic review and meta-analysis. Lancet 2009;373:1773-9.

7. Fadl $\mathrm{H}$, Magnuson A, Ostlund I, et al. Gestational diabetes mellitus and later cardiovascular disease: a Swedish population based case-control study. BJOG 2014;121:1530-6.

8. Alzaim M, Wood RJ. Vitamin $D$ and gestational diabetes mellitus. Nutr Rev 2013;71:158-67.

9. McCance DR. Diabetes in pregnancy. Best Pract Res Clin Obstet Gynaecol 2015;29:685-99.

10. Ben-Haroush A, Yogev Y, Hod M. Epidemiology of gestational diabetes mellitus and its association with Type 2 diabetes. Diabet Med 2004;21:103-13.

11. Hanna FWF, Peters JR. Screening for gestational diabetes; past, present and future. Diabet Med 2002;19:351-8.

12. Kruse AR, Darling M, Hansen $M$, et al. Recurrence of gestational diabetes in primiparous women. Acta Obs Gynecol Scand 2015;94:1367-72.

13. Schmidt Ml, Matos MC, Branchtein L, et al. Variation in glucose tolerance with ambient temperature. Lancet 1994;344:1054-5.

14. Akanji $\mathrm{AO}$, Bruce M, Frayn $\mathrm{K}$, et al. Oral glucose tolerance and ambient temperature in non-diabetic subjects. Diabetologia 1987;30:431-3.

15. Burris HH, Rifas-Shiman SL, Kleinman K, et al. Vitamin D deficiency in pregnancy and gestational diabetes mellitus. Am J Obstet Gynecol 2012;207:182.e1-8.

16. Clifton-Bligh RJ, McElduff $P$, McElduff $A$. Maternal vitamin $D$ deficiency, ethnicity and gestational diabetes. Diabet Med 2008;25:678-84.

17. Soheilykhah S, Mojibian M, Rashidi M, et al. Maternal vitamin D status in gestational diabetes mellitus. Nutr Clin Pract 2010;25:524-7.

18. Hossein-Nezhad A, Maghbooli Z, Vassigh A-R, et al. Prevalence of gestational diabetes mellitus and pregnancy outcomes in Iranian women. Taiwan J Obstet Gynecol 2007:46:236-41.

19. Zhang C, Qiu C, Hu FB, et al. Maternal plasma 25-hydroxyvitamin D concentrations and the risk for gestational diabetes mellitus. PLOS ONE 2008:3:e3753.

20. Parlea L, Bromberg IL, Feig DS, et al. Association between serum 25-hydroxyvitamin $D$ in early pregnancy and risk of gestational diabetes mellitus. Diabet Med 2012;29:e25-32.

21. Aghajafari F, Nagulesapillai T, Ronksley PE, et al. Association between maternal serum 25-hydroxyvitamin $D$ level and pregnancy and neonatal outcomes: systematic review and meta-analysis of observational studies. BMJ 2013;346:f1169.

22. Wei SQ, Qi HP, Luo ZC, et al. Maternal vitamin D status and adverse pregnancy outcomes: a systematic review and meta-analysis. J Matern Fetal Neonatal Med 2013;26:889-99.

23. Tobias Deidre K, Zhang C, van Dam RM, et al. Physical activity before and during pregnancy and risk of gestational diabetes mellitus: a meta-analysis. Diabetes Care 2011;34:223-9.

24. Tucker P, Gilliland J. The effect of season and weather on physical activity: a systematic review. Public Health 2007;121:909-22.

25. Watson PE, McDonald BW. Seasonal variation of nutrient intake in pregnancy: effects on infant measures and possible influence on diseases related to season of birth. Eur J Clin Nutr 2007:61:1271-80.

26. Lagunova Z, Porojnicu AC, Lindberg F, et al. The dependency of vitamin $D$ status on body mass index, gender, age and season. Anticancer Res 2009;29:3713-20.

27. Moses RG, Wong VCK, Lambert K, et al. Seasonal changes in the prevalence of gestational diabetes mellitus. Diabetes Care 2016;39:1218-21.

28. Australian Bureau of Statistics. Consensus of Population and Housing: Socio-Economic indexes for areas (SEIFA), Australia 2006. Data cube. Canberra. 2008, Rep No 2033055001 Contract No 2033055001.

29. Nelson W, Tong YL, Lee JK, et al. Methods for cosinor-rhythmometry. Chronobiologia 1979;6:305-23.

30. Moses R, Griffiths R. Is there a seasonal variation in the incidence of gestational diabetes? Diabet Med 1995;12:563-5.

31. Janghorbani M, Stenhouse E, Jones RB, et al. Gestational diabetes mellitus in Plymouth, U.K.: prevalence, seasonal variation and associated factors. J Reprod Med 2006;51:128-34. http://www.ncbi. nlm.nih.gov/pubmed/16572914 
32. Doro P, Benko R, Matuz M, et al. Seasonality in the incidence of type 2 diabetes. Diabetes Care 2006;29:173.

33. Barker DJP, Osmond C. Infant mortality, childhood nutrition, and ischemic heart disease in England and Wales. Lancet 1986;1:1077-81.

34. Barker DJP. The origins of the developmental origins theory. $J$ Intern Med 2007:261:412-17.

35. Vaiserman AM, Khalangot MD, Carstensen B, et al. Seasonality of birth in adult type 2 diabetic patients in three Ukrainian regions. Diabetologia 2009;52:2665-7.

36. Jensen CB, Zimmermann E, Gamborg M, et al. No evidence of seasonality of birth in adult type 2 diabetes in Denmark. Diabetologia 2015;58:2045-50.

37. Karamanos B, Thanopoulou A, Anastasiou E, et al. Relation of the Mediterranean diet with the incidence of gestational diabetes. Eur J Clin Nutr 2014;68:8-13.

38. Wei SQ, Audibert F, Luo ZC, et al. Maternal plasma 25-hydroxyvitamin $D$ levels, angiogenic factors, and preeclampsia. Am J Obstet Gynecol 2013;208:390.e1-6.
39. ADA. Gestational diabetes mellitus. Diabetes Care 2004;27(Suppl 1):S88-90.

40. Prasad M, Lumia M, Erkkola M, et al. Diet composition of pregnant Finnish women: changes over time and across seasons. Public Health Nutr 2010;13:939-46.

41. Holick MF. Vitamin D deficiency. N Engl J Med 2007;357:266-81.

42. Zhang MX, Pan GT, Guo JF, et al. Vitamin D deficiency increases the risk of gestational diabetes mellitus: a meta-analysis of observational studies. Nutrients 2015;7:8366-75.

43. Maestro B, Dávila N, Carranza MC, et al. Identification of a Vitamin $D$ response element in the human insulin receptor gene promoter. $J$ Steroid Biochem Mol Biol 2003;84:223-30.

44. Wang $\mathrm{Y}, \mathrm{He} \mathrm{D}, \mathrm{Ni} \mathrm{C}$, et al. Vitamin D induces autophagy of pancreatic $\beta$-cells and enhances insulin secretion. Mol Med Rep 2016;14:2644-50

45. Mclean A, Scott J, Keane RJ, et al. Validation of the 1994 South Australian perinatal data collection form. Adelaide, Australia: Pregnancy Outcome Unit, South Australian Department of Human Services, 2001. 